\title{
Neurophysiological and Spinal Cord Perfusion Changes at Dynamic Neck Positions in a Compressive Spinal Cord Injury Rat Model
}

\section{Zhengran $\mathrm{Yu}$}

Sun Yat-sen University First Affiliated Hospital

\section{Xing Cheng}

Sun Yat-sen University First Affiliated Hospital

Jiacheng Chen

Sun Yat-sen University First Affiliated Hospital

Sixiong Lin

Sun Yat-sen University First Affiliated Hospital

$\mathrm{HaO} \mathrm{Hu}$

Sun Yat-sen University First Affiliated Hospital

\section{Fangli Huang}

Sun Yat-sen University First Affiliated Hospital

\section{Zhiyuan Zou}

Sun Yat-sen University First Affiliated Hospital

\section{Bolin Chen}

Sun Yat-sen University First Affiliated Hospital

\section{Yong Wan}

Sun Yat-sen University First Affiliated Hospital

\section{Shaofu He}

Sun Yat-sen University First Affiliated Hospital

\section{Xinsheng Peng}

Sun Yat-sen University First Affiliated Hospital

Xuenong Zou ( $\square$ zxnong@hotmail.com )

Sun Yat-sen University First Affiliated Hospital https://orcid.org/0000-0002-8439-2624

\section{Research}

Keywords: chronic compressive spinal cord injury, dynamic neck positions, motor evoked potentials, somatosensory evoked potentials, spinal cord perfusion

Posted Date: April 13th, 2021 
DOI: https://doi.org/10.21203/rs.3.rs-404519/v1

License: (c) (1) This work is licensed under a Creative Commons Attribution 4.0 International License. Read Full License 


\section{Abstract}

Background: Cervical spondylotic myelopathy (CSM) is a degenerative condition of the spine that caused by static and dynamic compression of the spinal cord. However, the pathophysiological changes at dynamic neck positions remain poor. This study investigated the interplay between neurophysiological and haemodynamic responses at dynamic neck positions in a chronic compressive spinal cord injury (CCSCl) rat model.

Methods: Behavioural tests including Basso, Beattie, and Bresnahan scores and an inclined plane test were used to evaluate the motor function recovery. Combined examination of dynamic motor and somatosensory evoked potentials (DMEPs and DSSEPs, respectively) was performed regularly to evaluate the dynamic motor and sensory conduction of the cervical cord. At 4 weeks post-injury (wpi), dynamic magnetic resonance imaging (MRI) and dynamic laser Doppler flowmetry (LDF) were used to demonstrate the interstructure and spinal cord blood flow (SCBF) at the compression site at dynamic neck positions. Hematoxylin and eosin (HE) staining was performed to assess the cords' pathological changes.

Results: Behavioural tests and combined DMEPs and DSSEPs examination showed that spinal cord neurological function and dynamic neural conduction deteriorated gradually within a 4-week compression period. The DMEPs were mainly deteriorated upon flexion, while DSSEPs were upon all neck positions after the compression. At $4 \mathrm{wpi}$, dynamic MRI showed increased T2-weighted image (T2WI) signal intensities. Also, dynamic LDF demonstrated decreased SCBF at the spinal cord compression site. Both of them altered especially upon cervical flexion. The dynamic change in SCBF was significantly correlated with the change in DMEP amplitude upon flexion.

Conclusions: This exploratory study revealed that changes in axonal conduction in the motor and somatosensory tracts of the spinal cord were significantly related to chronic compression time and neck position. Furthermore, spinal cord ischaemia may be intimately related to motor conduction dysfunction upon flexion in $\mathrm{CCSCl}$ models. These results indicated the potential for therapies targeting dynamic spinal cord perfusion to prevent progression and functional loss in CSM.

\section{Introduction}

Cervical spondylotic myelopathy (CSM) is a degenerative condition of the spine that leads to static and dynamic compression of the spinal cord.[1] Dynamic compression of the spinal cord is recognized as an important pathogenic factor for CSM.[2] It has been proposed that dynamic injury may occur through instability,[3] an increase in the range of motion,[4] and minor trauma in the setting of pre-existing degenerative cervical myelopathy.[5]

Numerous clinical studies have reported the impacts of dynamic neck positions on CSM. Morphologically, Muhle et al.[6] used the dynamic magnetic resonance imaging (dynamic MRI) to demonstrate that the prevalence of spinal stenosis increased with flexion and extension compared with 
the prevalence in a neutral position. Further study reported extension shortens the cervical cord and decreases the space available for it with ligamentum flavum buckling [7], which could potentially aggravate posterior compression, causing pincer effects.[8, 9] On the other hand, although the spinal canal diameter may increase in flexion, some studies have reported that in this position, the tension forces applied to the spinal cord cause ventral spinal cord compression against osteophytes and discs, worsening eventual ventral compression.[8, 9] Neurophysiologically, CSM patients' dynamic somatosensory evoked potentials (DSSEPs) have been shown to deteriorate significantly upon extension and flexion. $[10,11]$ We further revealed that the percent changes in DSSEP amplitude at dynamic neck positions were related to preoperative radiographic characteristics, such as the presence of cervical segmental instability, compression degrees, and patterns of intramedullary T2WI hyperintensity.[12] Pathophysiological changes in the spinal cord at dynamic positions were demonstrated by cadaveric studies, in which the lateral columns and the anterior horns were deformed by mechanical stress produced by spondylotic bars during flexion.[8] Notably, the tissue of the spinal cord is highly vascularized and extremely sensitive to hypoxia induced by static or dynamic compression. The flexion position causes anterior compression of the cord, where the anterior spinal artery (ASA) traverses. Thus, it has also been suggested that ischaemia of the spinal cord as a result of neck flexion can cause neurological deteriorations and cervical myelopathies.[13-15] However, due to the lack of appropriate animal studies, the mechanisms behind the changes in neurological functions at dynamic neck positions are still not clear.

In recent years, numerous attempts have been made to establish a CSM in cases of chronic compressive spinal cord injury (CCSCl) model in different animals, employing various methods, such as tumour induction[16], the use of penetrating hydrogels[17] or urethane polymers[18], the use of spinal hyperostotic mice (twy/twy)[19], and plastic screw implantation.[20] Among these approaches, hydrogels and polymers are expected to be applicable materials that lead to chronically progressive injury. Previously, we successfully established a CCSCl rat model using a water-absorbable polyurethane polymer sheet[21], which provided the foundation for this study. We have found that various changes, including decreased microvascular density,[22, 23] varying degrees of ischaemia-hypoxia,[24, 25] BSCB disruption,[25,26] and neuron loss,[27] occurred at different time points after static chronic spinal cord compression. Nevertheless, neurophysiological changes and their relationship with perfusion alterations at dynamic neck positions were remained largely unknown. In this study, we used $\mathrm{CCSCl}$ rat models to study the changes in dynamic MRI, dynamic motor evoked potentials (DMEPs), DSSEPs, and spinal cord blood flow (SCBF) at different neck positions and further analysed the correlations between them.

\section{Materials And Methods}

\section{Study design}

All experimental procedures were approved by the Research Ethics Committee of Sun Yat-sen University, Guangzhou, China and conformed to all relevant regulatory standards. A total of 46 male adult SpragueDawley (SD) rats $(250-400 \mathrm{~g})$ were randomly allocated to the sham $(n=16)$ and $\operatorname{CCSCl}(n=30)$ groups. 
The animals in the CCSCl group underwent implantation of a water-absorbing polymer sheet into the cervical spinal canal, which expanded over time to induce chronic compression of the cord. Neurological functions were evaluated by behavioural tests from 1 day post-injury (dpi) to 4 weeks post-injury (wpi), and DMEPs and DSSEPs tests from 1 to 4 wpi. At 4 wpi, the structural changes of the cord at various neck positions were evaluated by dynamic MRI scans. The perfusion status of the cord at dynamic positions was assessed by laser Doppler flowmetry (LDF). The ultrastructure of the cord was evaluated by routine histology. (Fig. 1A)

\section{Induction of spinal cord chronic compression}

Each rat in the sham and $\mathrm{CCSCl}$ groups was anaesthetized with $10 \%$ chloral hydrate $(300 \mathrm{mg} / \mathrm{kg})$ (Guangzhou FISCLAB Environ. Sci-Tech. Co., Ltd., Guangzhou, China). Following exposure of the spinal process and laminae of $\mathrm{C} 4-\mathrm{C} 6$ from the posterior, the ligamentum flavum and $\mathrm{C} 5$ lamina were removed to access the epidural space. In the CCSCI group, the polymer (1 $\rightarrow 4)-3,6$-anhydro-a-I-galactopyranosyl$(1 \rightarrow 3)-\beta$-D-galactopyranan) sheet $(1 \mathrm{~mm} \times 3 \mathrm{~mm} \times 1 \mathrm{~mm}$ ) was implanted into the $\mathrm{C} 6$ epidural space on the dorsal part of the spinal cord. Spinal cord compression was achieved by expansion of the polymer caused by liquid absorption.[22, 28] This polymer sheet can absorb liquid in the spinal canal to expand its volume sevenfold (approximately $2.3 \mathrm{~mm} \times 4.2 \mathrm{~mm} \times 2.2 \mathrm{~mm}$ ). In the sham group, the C5 lamina was removed without insertion of the polymer sheet. Following surgery, the incision was closed in layers with complete haemostasis. To prevent dehydration, animals received a subcutaneous (s.c.) injection of lactated Ringer's solution $(200 \mu \mathrm{L})$ immediately after surgery. All rats were administered an intramuscular injection of penicillin $\mathrm{G}(80 \mathrm{U} / \mathrm{g})$ during surgery to prevent infection, and carprofen $(4-5 \mathrm{mg} / \mathrm{kg}$, Rimadyl, Pfizer) was injected subcutaneously 2 days post-surgery for further pain relief as needed. All surgeries were performed by the same experienced investigator.

\section{Neurological Function Evaluation}

To evaluate motor function recovery after $\mathrm{CCSCl}$, the Basso, Beattie, and Bresnahan (BBB) locomotor scale[29] and an inclined plane test (IPT)[30] were conducted at 1 dpi and 1, 2, 3 and 4 wpi. BBB scores ranged between 0 and 21; a score of 0 reflected complete paralysis and a score of 21 indicated normal locomotion. Lower scores ( $0-7)$ denoted isolated joint movements with little or no hindlimb movement; intermediate scores (8-13) indicated intervals of uncoordinated stepping; and higher scores (14-21) signified forelimb and hindlimb coordination. For the IPT, rats were placed horizontally on a smooth, tilted board. The board was initially placed in a horizontal orientation $\left(0^{\circ}\right)$, and the angle of the board was increased by $5^{\circ}-10^{\circ}$ after each attempt. The maximum angle at which the rats remained on the board for 10 seconds was recorded. The evaluation was conducted by two investigators blinded to the group assignments.

\section{Histological evaluations}

In brief, rats in different groups were euthanized with an overdose of intravenous sodium pentobarbital and transcardially perfused with $0.9 \%$ saline followed by $4 \%$ paraformaldehyde in $0.1 \mathrm{M}$ phosphate 
buffer (PFA) at the endpoint of the study. The C5-C7 spinal cord was harvested, fixed overnight with $4 \%$ formaldehyde in phosphate-buffered solution at $4^{\circ} \mathrm{C}$, and embedded in paraffin. A series of $25-\mu \mathrm{m}$ thick spinal cord sections were used for immunohistochemical staining. Slides were placed on a plastic rack and vessel for microwave epitope retrieval.

\section{Electrophysiological evaluations}

The functional integrity of the spinal cord among the model rats at dynamic positions was evaluated by DMEPs and DSSEPs at 1 to 4 wpi. An electrophysiological monitoring system (Nicolet Endeavor CR) was used to elicit and record transcranial electrical stimulation motor evoked potentials (TES-MEPs) and SSEPs. The animals were evaluated under general anaesthesia with $10 \%$ chloral hydrate $(300 \mathrm{mg} / \mathrm{kg})$ (Guangzhou FISCLAB Environ. Sci-Tech. Co., Ltd., Guangzhou, China) intraperitoneally. Their scalps and the posterior portion of their necks were shaved and aseptically treated using iodine. Two electrodes were placed $2 \mathrm{~mm}$ posterior to bregma and $3 \mathrm{~mm}$ to the left and right of the longitudinal midline; these locations correspond to the left or right primary motor cortex (C3 and C4), respectively, and served as DMEP stimulation electrodes and a reference electrode alternatively. Another two electrodes were placed at the midline of the skull $2 \mathrm{~mm}$ anterior to bregma $(\mathrm{Fz})$ and at the midpoint of the ears $(\mathrm{Cz})$ for DSSEP recording. Two electrodes were placed around each side of the sciatic nerve along the course of the biceps femoris muscle for DSSEP stimulation. Two were placed at each side of the extensor digitorum communis in the forelimb and tibialis anterior in the hindlimb for DMEP recording. A ground electrode was placed on the back of the subject subcutaneously. Rats were positioned neutrally at approximately $40^{\circ}$ extension and then at approximately $40^{\circ}$ flexion of the cervical spine using an angle-adjustable stereotaxic frame for dynamic MEP and SSEP measurements. (Fig. 3A and Fig. 4A)

Constant current stimulation at the skull was used for the generation of MEPs. Single-trial MEPs were obtained with a current intensity of up to $16 \mathrm{~mA}$ and a pulse width of $50 \mathrm{ls}$ at a frequency of $350 \mathrm{~Hz}$ for a 1-minute duration. MEPs were recorded using subdermal needle electrode pairs from the extensor digitorum communis in the forelimb and tibialis anterior in the hindlimb.

Constant current stimulation around sciatic nerves with a magnitude of $6 \mathrm{~mA}$, duration of $0.02 \mathrm{~ms}$, and frequency of $3.43 \mathrm{~Hz}$ was used to elicit DSSEPs. Cortical SSEPs were recorded from the skull at Cz-Fz. We averaged 256 SSEP trials to improve the signal-to-noise ratio. SSEP signals were filtered using a bandpass filter of $10 \mathrm{~Hz}$ to $250 \mathrm{~Hz}$. A sensitivity of $20 \mathrm{IV} /$ div and a time base of $5 \mathrm{~ms} /$ div were used to display the SSEP responses.

Onset latency and peak-to-peak amplitude of the responses at dynamic positions were measured at the endpoint of the study. The onset latency was measured from the delivery of the stimulus to the first positive or negative deflection (N1 in Fig. 4) from baseline. Peak-to-peak amplitude was defined as the maximum amplitude between the largest positive and negative peak (N1-P2 in Fig. 4). Each MEP/SSEP test was repeated 3 times, and their average value was taken. We defined an immeasurable SSEP/MEP as a waveform that could not be identified by averaging over 500 sweeps. SSEP and MEP responses 
recorded from each limb were classified separately. For the final analysis, the lowest SSEP or MEP amplitude among the four limbs was utilized as the definitive data point.

\section{MRI evaluations}

Cord compression was evaluated by MRI using a 3.0-T MR imager (Siemens Trio). The animals were anaesthetized by isoflurane inhalation, and a surface coil was placed over the animals' cervical spine region to acquire anatomical T2-weighted images (T2WIs). T2Wls were acquired with the following parameters: echo time[31]/repetition time $(T R)=35 / 2500 \mathrm{~ms}(T 2 W)$ and $115 / 2500 \mathrm{~ms}$ (PDW), slice thickness $=1 \mathrm{~mm}$, interslice distance $=1.1 \mathrm{~mm}$, and number of excitations $(\mathrm{NEX})=4$. A total of 15 axial slices covering $\mathrm{C} 3-\mathrm{C} 7$ of the cervical spinal cord were acquired at each disc and body level. The image slice planning was the same as that in anatomical axial images, with 15 slices covering the cervical spinal cord from $\mathrm{C} 3$ to $\mathrm{C} 7$.

The sagittal diameters of the spinal canals and spinal cords and the transverse diameters of the cords and canals were measured with Osirx (Pixmeo, Geneva, Switzerland), which is a standard software for the MR equipment packages. MR signal intensity was analysed in regions of interest (ROIs) at the compression site (or C6 levels in the sham group) of the spinal cord with Osirx. We used the T2WI intensity signals of paraspinal muscles as calibration references in each MR image and quantified the relative signal intensities of the $\mathrm{ROI}$ in cervical grey matter in both sham and $\mathrm{CCSCl}$ rats. The relative signal intensity was calculated by dividing the signal intensity of the ROI in cervical grey matter by that in paraspinal muscles.

\section{Dynamic LDF measurements}

We measured the SCBF and oxygen saturation $\left(\mathrm{SO}_{2}\right)$ of the rats at 10 minutes and 4 weeks after implanting the compression material in the $\mathrm{CCSCl}$ group or exposing the dorsal dura mater in the sham group. For SCBF and $\mathrm{SO}_{2}$ measurements, an OxyFlo (ADInstruments Pty Ltd, Castle Hill, Australia) LDF probe was attached to the stereotaxic frame and positioned in contact with the dorsal dura mater to the right side of the central vein at the $\mathrm{C} 3$ and $\mathrm{C} 6$ segmental levels of the spinal cord. Upon neutral positioning, after adjusting the SCBF at $\mathrm{C} 3$ to the same position (580 PU) for both the sham and $\mathrm{CCSCl}$ models, we monitored the SCBF at the $\mathrm{C} 6$ (compression) level in the sham and $\mathrm{CCSCl}$ models. The rats were then positioned upon maximum cervical extension and cervical flexion postures for at least 5 minutes before undergoing the same recording process. Each recording process started with an adjustment of the SCBF data at the $\mathrm{C} 3$ level, and the recording time at $\mathrm{C} 6$ or the injury level lasted for at least 1 minute. Output signals were recorded in Powerlab (ADInstruments Pty Ltd, Castle Hill, Australia) continuously throughout the experiment and averaged every 3 seconds.

\section{Statistical analysis}

Comparisons of the ultrastructure of the spinal cord on the injured and non-injured sides were performed using paired t-tests. Comparisons of the neural conduction situations of spinal cords among the animals categorized by DMEP and DSSEP responses and spinal cord perfusion status demonstrated by LDF at 
different neck positions were performed using one-way analysis of variance (ANOVA) and post hoc tests. The level of significance was set at $p<0.05$. All data analyses were performed using SPSS 15.0 analysis software (SPSS Inc., Chicago, IL, USA).

\section{Results}

\section{Neurological dysfunction after the induction of CCSCI}

Following incomplete CCSCl, progressive neurological deterioration occurred in a sizable number of patients; similar results have also been observed in animal models.[32] Here, we used the BBB score and IPT outcome to evaluate the changes in neurological function in a rat model of CCSCl. In our study, from $1 \mathrm{dpi}$ to $4 \mathrm{wpi}$, the BBB scores and IPT angles of the $\mathrm{CCSCl}$ rat models gradually decreased and were significantly lower than those of the sham models after one and two wpi. (Fig. 1B, C)

\section{Structural and cellular characteristics in sham and CCSCI spinal cords}

Haematoxylin and eosin (HE) staining revealed histological changes at the injury epicentre under different levels of magnification (Fig. 2). The spinal cord was intact in the sham group. Polygonal Nissl bodies inside neurons in the grey matter anterior horn were large and dense. In a transverse section of a $\mathrm{CCSCl}$ model, the compressed spinal cord exhibited structural damage, including fragmentation of neuronal nuclei, pyknosis, neuropil damage, degradation of the extracellular matrix, interstitial oedema, cytoplasmic reduction, and cavity formation. The dorsal funiculi suffered the most severe breakage. Neuronal cell bodies in the anterior horn were round and small. Nissl body chromatolysis was evident inside the neurons. The insults to the cord circulation in the $\mathrm{CCSCl}$ models included central canal enlargement and intratissue bleeding in the grey matter. The longitudinal neurological conduction pathways of the $\mathrm{CCSCl}$ models were severely disrupted, as neuronal loss in the grey and axon breakage in the white matter were observed in sagittal sections.

\section{Dynamic motor deficits in CCSCI models evaluated by DMEPs}

DMEPs were tested at 1, 2, 3 and 4 wpi, following the protocol described in the Methods section. The number of rats with abolished waves and the latency and amplitude data of the remaining rats are summarized in Table 1. The latency and amplitude of DMEP peaks in the sham group at all three positions remained unchanged at all testing time points. After chronic compression injury, the MEP response was abolished in one rat at all neck positions. At the first $2 \mathrm{wpi}$, the average DMEP latencies and amplitudes of the CCSCI models were slightly affected upon neutrality and extension but were significantly diminished upon flexion (t-test, $p<0.001$ ) compared with the average DMEP latencies and amplitudes of the sham models. At $4 \mathrm{wpi}$, the DMEP latencies and amplitudes of the CCSCl rats were significantly diminished at all neck positions compared with the average DMEP latencies and amplitudes 
of the sham rats. It should be noted that the CCSCl models had the worst DMEP latencies and amplitudes (ANOVA post hoc test, $p<0.001$ ) and the most abolished DMEP waves (Chi-square, $p<0.05$ ) upon flexion compared with the values at the other two positions at all time points after compression. (Fig. 3) 
Table 1

DMEPs in Sham and CCSCl rat models after compression injury

Sham

Neutral
Extension Flexion

$1 \mathrm{Wpi}$

Latency (ms)

$7.96 \pm$

$8.08 \pm$

1.07

$8.54 \pm$

1.26

Amplitude $(\mu \mathrm{V})$

$395.67 \pm$ 45.61

$380.48 \pm$

43.59

$385.27 \pm$

36.78

0

0

$\mathrm{ccscl}$

Immeasurable $\quad 0$

waves(n)

$2 \mathrm{Wpi}$

Latency(ms)

$7.98 \pm$

1.24

$7.99 \pm$

$7.73 \pm$

1.31

Amplitude( $\mu \mathrm{V})$

$374.84 \pm$ 43.74

$399.4 \pm$

35.67

$390.12 \pm$

34.88

0

Immeasurable

waves(n)

0

$3 \mathrm{Wpi}$

Latency(ms)

$7.74 \pm$

1.21

$8.01 \pm$

1.39

$7.79 \pm$

$9.42 \pm$

$8.33 \pm 1.55$

$386.98 \pm$

28.38

$8.31 \pm 1.16$

$8.52 \pm 1.54$

Extension

Flexion

Neutral

1

$382.56 \pm$

23.84

$334.19 \pm$

$26.56^{\# \star \star \star \star}$

4

$\begin{array}{lllllll}\text { Amplitude }(\mu \mathrm{V}) & 381.25 \pm & 392.4 \pm & 375.78 \pm & 368.21 \pm & 367.77 \pm & 234.75 \pm \\ & 38.86 & 43.88 & 49.74 & 29.23 & 22.35 & 31.12^{\# \star \star \star}\end{array}$

Immeasurable $\quad 0 \quad 0 \quad 00$

waves(n)

$4 \mathrm{Wpi}$

\begin{tabular}{|c|c|c|c|c|c|c|}
\hline Latency (ms) & $\begin{array}{l}7.83 \pm \\
1.34\end{array}$ & $\begin{array}{l}7.52 \pm \\
1.19\end{array}$ & $\begin{array}{l}7.95 \pm \\
1.42\end{array}$ & $\begin{array}{l}9.61 \pm \\
2.33^{\star}\end{array}$ & $\begin{array}{l}9.42 \pm \\
1.84^{\star}\end{array}$ & $\begin{array}{l}12.57 \pm \\
2.68^{\#_{\star}}\end{array}$ \\
\hline Amp & $\begin{array}{l}392.66 \pm \\
47.71\end{array}$ & $\begin{array}{l}389.42 \pm \\
46.56\end{array}$ & $\begin{array}{l}398.58 \pm \\
39.94\end{array}$ & $\begin{array}{l}345.3 \pm \\
34.43^{\star \star \star}\end{array}$ & $\begin{array}{l}353.85 \pm \\
31.19 * *\end{array}$ & $\begin{array}{l}215.39 \pm \\
18.82^{\#_{\star \star \star \star}}\end{array}$ \\
\hline
\end{tabular}

$*, * *, * * *$ Indicated statistical significance $(p<0.05,0.01,0.001$ respectively) in the difference between the Sham and CCSCl group using t test.

\# Indicated statistical significance $(p<0.05)$ in the difference between various neck positions MEP responses using one-way ANOVA and post hoc test. 


\begin{tabular}{|c|c|c|c|c|}
\hline & \multicolumn{2}{|l|}{ Sham } & \multicolumn{2}{|l|}{$\mathrm{ccscl}$} \\
\hline $\begin{array}{l}\text { Immeasurable } \\
\text { waves(n) }\end{array}$ & 0 & 0 & 4 & $13^{\#}$ \\
\hline \multicolumn{5}{|c|}{$\begin{array}{l}*, * *, * * * \text { Indicated statistical significance }(p<0.05,0.01,0.001 \text { respectively) in the difference between } \\
\text { the Sham and CCSCl group using } t \text { test. }\end{array}$} \\
\hline \multicolumn{5}{|c|}{$\begin{array}{l}\text { \# Indicated statistical significance }(\mathrm{p}<0.05) \text { in the difference between various neck positions MEP } \\
\text { responses using one-way ANOVA and post hoc test. }\end{array}$} \\
\hline
\end{tabular}

After a few minutes of testing the DMEPs, DSSEPs of rat all models were tested. The number of rats with abolished waves and the latency and amplitude data are summarized in Table 2. The latency and amplitude of DMEP peaks in the sham group at all three positions remained unchanged at all testing time points. From 1 to 4 weeks of chronic compression injury, increasing numbers of rats showed abolished SSEPs with increasing compression time. The CCSCI models had more abolished DSSEP waves and significantly lower DSSEP amplitudes than the sham models at all neck positions at each time point after injury (t-test, $\mathrm{p}<0.001$ ). At the first $2 \mathrm{wpi}, \mathrm{CCSCl}$ rats had fewer abolished waves and significantly higher (ANOVA post hoc test, $p<0.05$ ) DSSEP amplitudes at neutral positions than at the other two neck positions. The DSSEP amplitudes at all 3 neck positions were similar after 3 wpi. (Fig. 4) 
Table 2

DSSEPs in Sham and CCSCl rat models after compression injury

Sham

Neutral Extension Flexion Neutral Extension Flexion

$1 \mathrm{Wpi}$

Latency (ms)

$15.24 \pm$

2.81

$15.97 \pm$

3.47

$16.11 \pm$

3.55

$16.43 \pm$

3.22

$17.19 \pm$

3.06

Amplitude $(\mu \mathrm{V})$

$21.66 \pm$

$21.99 \pm$

$21.23 \pm$

$18.25 \pm$

$1.85^{\star \star \star}$

$15.13 \pm$

$0.79^{\#_{\star \star \star \star}}$

2

7

Immeasurable $\quad 0$

waves(n)

$2 \mathrm{Wpi}$

Latency (ms)

$15.68 \pm$

2.32

$15.94 \pm$

3.28

$16.42 \pm$

3.17

$15.94 \pm$

3.02

$18.26 \pm$

3.41

Amplitude $(\mu \mathrm{V})$

$22.03 \pm$
1.25

$22.54 \pm$

1.17

$21.63 \pm$

$17.87 \pm$

$1.32^{\star \star \star}$

$14.57 \pm$

$1.07^{\# \star \star \star}$

4

11

0

waves(n)

$3 \mathrm{Wpi}$

Latency (ms)

$16.24 \pm$

3.67

0

0

4

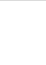

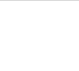

11

1

$17.71 \pm$

4.37

2.89

$13.3 \pm$

$1.52^{\star \star \star}$

11

Immeasu
waves(n)

$4 \mathrm{Wpi}$

Latency (ms)

$15.99 \pm$

$15.64 \pm$

$16.05 \pm$

$17.56 \pm$

4.83

Amplitude $(\mu \mathrm{V})$

$22.03 \pm$

1.32

2.71

2.77

$12.49 \pm$

$1.4^{\star \star \star}$

$21.8 \pm$

1.12

1.43

16

0

waves(n)

$0 \quad 0$

$0 \quad 0$

(n)
$17.93 \pm$

4.79

$12.84 \pm$

$0.86 * \star \star$

13

14

13

3

$18.32 \pm 5.4 \quad 16.3 \pm 5.11$

$16.21 \pm 6.72$
$15.08 \pm$

$1.09^{\# \star \star \star}$

3

$13.59 \pm$

$2.06^{\# \star \star \star}$

7

$16.33 \pm 2.58$

$17.33 \pm 2.95$

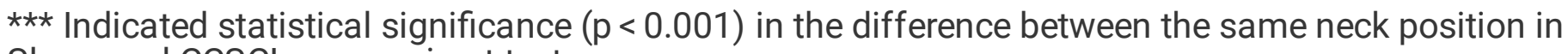
Sham and $\mathrm{CCSCl}$ group using $\mathrm{t}$ test.

\# Indicated statistical significance $(p<0.05)$ in the difference between various neck positions MEP responses using one-way ANOVA and post hoc test. 


\section{Dynamic MRI showed intramedullary T2WI hyperintensity of the spinal cord upon flexion}

To explore the inner structural changes in $\mathrm{CCSCl}$ rat models at dynamic neck positions, we performed dynamic MRI on the cervical cord region of our models at 4 wpi. Compared with the sham model, compression was clearly evident at the posterior side of the spinal cord at the $\mathrm{C} 6$ region of $\mathrm{CCSCl}$ rat models (Fig. 5a-h). The sagittal diameter of the spinal canal $(1.89 \pm 0.11 \mathrm{~mm})$ and spinal cord $(0.97 \pm$ $0.06 \mathrm{~mm}$ ) in the CCSCl group was significantly smaller than that (canal: $2.85 \pm 0.09 \mathrm{~mm}$, cord: $2.10 \pm 0.04$ $\mathrm{mm}$ ) in the sham group (t-test, $\mathrm{p}<0.001$ ). The sagittal diameter of the canal decreased $27.3 \%$ and the cord decreased $53.7 \%$ in $\mathrm{CCSCl}$ group compared with the sham group. The transverse diameters of the canal were similar in the $\mathrm{CCSCl}$ and sham groups (pooled mean $5.42 \pm 0.06 \mathrm{~mm}$ ). The spinal canal and cord diameters did not vary significantly among all three neck positions in either the CCSCl or sham group.

We then analysed the relative T2WI signal intensities of the ROI in cervical grey matter in both sham and $\mathrm{CCSCl}$ rats. There was a significantly higher T2WI contrast medium intensity at the injured site in the neutral position in CCSCl rats (sagittal: $2.07 \pm 0.16$, transverse: $2.05 \pm 0.19$ ) than in sham rats (sagittal: $1.45 \pm 0.14$, transverse: $1.89 \pm 0.13$ ) (t-test, sagittal: $p<0.001$, transverse: $p=0.002$ ). T2WI intensity was even greater at the injured site of $\mathrm{CCSCl}$ rats in the flexion position (sagittal: $3.17 \pm 0.17$; transverse: 2.61 \pm 0.15 ) than in the neutral (sagittal: $2.07 \pm 0.16$, transverse: $2.05 \pm 0.19$ ) or extension positions (sagittal: $2.15 \pm 0.15$, transverse: $2.12 \pm 0.13$ ) (t-test, $p<0.001$ ). (Fig. 5i, j)

\section{Dynamic LDF showed decreased perfusion of the spinal cord upon flexion}

The LDF data gave an overview of perfusion at the injury site and made it accessible for comparative analysis of SCBF among different locations. At 10 minutes after compression injury, the SCBF and $\mathrm{SO}_{2}$ were similar between the sham and $\mathrm{CCSCl}$ models, indicating that there were hardly any effects of the unexpanded material on the perfusion of the cord at all neck positions (Supplementary Fig. S1). At 4 wpi, the SCBF (pooled mean 572.69 $\pm 19.29 \mathrm{PU}$ ) and $\mathrm{SO}_{2}$ (pooled mean $91.29 \pm 1.71 \%$ ) in sham models remained unchanged in all three neck positions. For the $\mathrm{CCSCl}$ models, their $\mathrm{SCBF}$ and $\mathrm{SO}_{2}$ at all three neck positions (neutral: $\mathrm{SCBF}=382.6 \pm 29.81 \mathrm{PU}, \mathrm{SO}_{2}=70.63 \pm 4.37 \%$; extension: $\mathrm{SCBF}=372.83 \pm 24.81$ $\mathrm{PU}, \mathrm{SO} 2=67.13 \pm 8.43 \%$; flexion: $\mathrm{SCBF}=213.47 \pm 25.55 \mathrm{PU}, \mathrm{SO} 2=39.27 \pm 4.97 \%$ ) decreased significantly (t-test, $\mathrm{p}<0.001$ ) compared with the sham models. Furthermore, while the $\mathrm{SCBF}$ and $\mathrm{SO}_{2}$ did not change significantly from neutral to extension positions, both of these measures decreased significantly upon flexion in the CCSCI models (t-test, $\mathrm{p}<0.001$ ). (Fig. 6a-e) We also calculated the relative change in SCBF (R-SCBF) and oxygen saturation ( $\mathrm{R}-\mathrm{SO}_{2} \%$ ) upon extension or flexion by dividing each model's SCBF and $\mathrm{SO}_{2} \%$ at extension or flexion by that at the neutral position. The R-SCBF at $4 \mathrm{wpi}$ of $\mathrm{CCSCl}$ rat models were $0.98 \pm 0.14$ and $0.73 \pm 0.11$ upon extension and flexion respectively. The $\mathrm{R}-\mathrm{SO}_{2} \%$ at 4 wpi of $\mathrm{CCSCl}$ rat models were $0.96 \pm 0.15$ and $0.56 \pm 0.09$ upon extension and flexion respectively. Both the R-SCBF and 
$\mathrm{R}-\mathrm{SO}_{2} \%$ at flexion were significantly lower than that at extension (t-test, $\mathrm{p}<0.001$ ) for the CCSCI model at 4 wpi. (Supplementary Fig. S2)

\section{Correlations between dynamic neurophysiological and LDF changes in the spinal cord}

We calculated the relative amplitudes of DMEPs (RA-DMEP) and DSSEPS (RA-DSSEP) upon extension or flexion by dividing each model's DMEP and DSSEP amplitude upon extension or flexion by that at neutral position. The immeasurable waveforms were also included with their amplitudes defined as $0 \mu \mathrm{V}$. The RADMEPs gradually decreased upon flexion and were significantly lower than those upon extension at each time point after injury (t-test, $\mathrm{p}<0.05$ ), while the RA-DSSEPs did not vary significantly upon extension and flexion. (Supplementary Fig. S3) Correlation analysis found that the R-SCBF was significantly correlated with the RA-DMEP (Spearman correlation, $R=0.72, p<0.001$ ) upon flexion, while there was no correlation between them upon extension. Neither the correlations between R-SCBF and RA-DSSEP at extension and flexion were significant. (Fig. 6f-i)

\section{Discussion}

This study focused on the interaction between neurophysiological and haemodynamic responses at dynamic neck positions after CCSCI. The present study clearly demonstrates that the behavioural assessment, DSSEPs and DMEPs deteriorated with compression time and different neck positions. At 1 and 2 wpi, DSSEPs were negatively affected at all neck positions and were significantly deteriorated at both extension and flexion, while the DMEPs were only affected at the flexion position. After 3 wpi, DSSEPs were all severely reduced and were identical at all neck positions. The DMEPs were always significantly deteriorated upon flexion after the compression, but were not damaged significantly upon neutrality and extension until 4 wpi. Dynamic MRI data at 4 wpi revealed that the sagittal diameter of the canal was compromised by more than a quarter (26.3\%) and the spinal cord by more than half (53.7\%) in CCSCI models and did not vary among different postures. The T2WI signal intensities of CCSCI models' cervical grey matter at flexion were significantly greater than those at neutral and extension, suggestive of severer ischemia at flexion. So we looked into the CCSCI models' perfusion at the compression site, and found it was significantly deteriorated at 4 wpi, especially upon cervical flexion. The dynamic change in SCBF was significantly correlated with the change in DMEP and DSSEP amplitudes upon flexion. These results could provide new insights for the development of drug therapy targeting spinal cord perfusion to prevent the progression and functional loss of CSM.

SSEPs and MEPs are distinct modalities that can be used to monitor the spinal cord. SSEPs reflect axonal conduction in the ascending sensory tracts in the posterior columns. MEPs monitor the descending motor system located in the anterior and lateral corticospinal tracts and the anterior horn motor neuron system, including the function of ischaemia-sensitive a motor neurons. The different conductive pathways of SSEPs and MEPs result in different characteristics of SSEPs and MEPS as indicators of spinal cord function, with respect to spinal cord ischaemia response time and false-positive 
and false-negative results.[33] Ueta et al.[34] investigated the effects of focal compression on physiologic integrity; electrophysiological manifestations including MEPs, SEPs and spine-to-spine potentials, spinal cord circulation and clinical status in four different directions of compression in pigs. Fehlings et al. reported the characteristics of MEP and SEP changes,[35] as well as their relationships with the severity of the injury and the SCBF[36] in spinal cord injury rat models. Lee et al.[37] and Hu et al.[38] reported SEP changes in two different CSM rat models using titanium screws and water-absorbable polyurethane polymers. Here, we used a well-established CCSCI rat model, in which the cervical cord was compressed by a gradually expanding water-absorbable polyurethane polymer.[39] The SCBF did not show deficits immediately after compression, and BBB scores and IPT tests were normal at 1 day after surgery, indicating that unexpanded compression material could be placed into the epidural space of the canal with no harm. The CCSCI models developed significant behavioural dysfunction, demonstrated by a significant drop in BBB scores and IPT angles after 1 wpi, which was in line with other studies.[32, 40] In addition, we detected significant DSSEP changes rather than DMEP changes upon a neutral neck position at $1 \mathrm{wpi}$, indicating early somatosensory tract disruption. DSSEP deterioration was more severe upon extension and milder upon a neutral position, suggesting that it is dynamically reversible. DMEPs showed significant deterioration upon flexion and remained relatively unchanged at neutral and extension at 1 wpi, indicating that dorsal compression disrupted the motor conduction pathway only in the flexed position. After $3 \mathrm{wpi}$, the DSSEP severely deteriorated at all neck positions, including neutral positions, indicating that the recuperation capability decreased. Until 4 wpi, the DMEP at neutral and extension positions also showed significant abnormalities compared with the sham group but was still significantly better than that at the flexion position. We assume this was because dorsal compression had much less influence on the anterior horn and motor conduction pathways. CSM patients suffering from hypertrophic ligamentum flavum generally present ataxia, which is mainly caused by dorsal funiculus disruption, but present full strength in all 4 extremities.[41] Ueta et al.[34] noted that SEPs were lost first during posterior, circumferential, and lateral acute transient compressions, while MEPs were lost first during anterior compression in pigs, which coordinated with our findings.

To better understand the mechanisms of varied DMEP and DSSEP performance at dynamic neck positions, we performed dynamic MRI of both sham and $\mathrm{CCSCl}$ rats. The relative $\mathrm{T} 2 \mathrm{WI}$ signal intensities of the ROI in cervical grey matter in both the sham and $\mathrm{CCSCl}$ rats were analysed. The T2WI intensity was the greatest upon flexion compared with that at neutral or extension positions. This finding was in line with many clinical studies.[42, 43] While some authors assumed that cervical canal expansion at flexion permits better visualization of intramedullary hyperintensity on $\mathrm{T} 2 \mathrm{WI},[42,43]$ others concluded that hyperintensities were more related to spinal cord ischaemia.[44] The significance of these lesions in CSM patients is also controversial in the literature.[45] Some studies have reported that the pattern of intramedullary hyperintensity lesions is an important criterion. $[46,47]$ In this study, the spinal canal remained constant at all three positions; also, we found a significant hyperintense pencil-like lesion pattern on sagittal T2WIs and the so-called "snake eyes" or "owl's eyes pattern" with hyperintense signal conversion on axial T2WIs of CCSCI models upon flexion (see Fig. 5D, H). Previous studies reported that these typical "pencil-like" lesions on sagittal orientation and "snake eyes" lesions on axial orientation were 
important MRI characteristics of spinal cord infarction, indicating partially symmetrical small infarcts in the anterior horns.[44, 48] Intramedullary T2WI hyperintensity represents a variety of histologic changes, including oedema, ischaemia, demyelination, gliosis, microcavities, and cavities. Similarly, histological analysis in this study showed that the compressed spinal cord exhibited structural damage in its anterior horn, including fragmentation of neuronal nuclei, pyknosis, neuropil damage, degradation of the extracellular matrix, interstitial oedema, cytoplasmic reduction, and cavity formation. It has been reported that under chronic focal spinal cord compression, there was a decrease in blood flow in the compressed segment, which contributed to the pathogenesis of myelopathy.[18] Our previous study showed that 4 weeks of chronic compression led to a severe ischaemia-hypoxia environment, as demonstrated by increased HIF-1 a expression,[25] and significantly decreased microvascular density, as demonstrated by micro-CT and immunohistochemistry data. $[39,49]$ Thus, the increased T2WI hyperintensity in our study was more likely to represent transient ischaemia upon flexion. We assume that the CCSCI models, whose spinal cord circulation was already compromised, suffered more perfusion decrease upon flexion than sham mice. The increased T2WI hyperintensity at flexion in CCSCl models is suggestive of a transient decrease in blood perfusion of the cord, which still needs to be further confirmed.

LDF is a non-invasive method for blood flow measurement, which makes it preferable for measuring microcirculatory alterations of the spinal cord at different neck positions. The technique uses tissue backscattered light to qualitatively assess the blood flow rate and has been a useful method for quantifying perfusion of the spinal cord in several animal and human studies.[50-52] Brain et al.[53] first reported ischemia as one of the crucial pathophysiological mechanisms in CSM. Pathological changes such as vessel wall thickening and hyalinization in the anterior spinal artery and parenchymal arterioles[53], and decreased number of vessels as indicated by the decreased laminin staining have been identified in chronic compressed cervical cords[54]. Kurokawa et al[18] previously reported an approximately $20 \%$ reduction in blood flow in the compressed segment (C5-C6) compared with that of the rostral (C3-C4) segment in $\mathrm{CCSCl}$ rat models using hydrogen clearance to measure blood flow. In our study, the reduction value was $33 \%$ in neutral position. The SCBF data in the two studies were not comparable because Kurokawa et al[18] did not quantify their compression degree. Moreover, we firstly report a $27 \%$ reduction of SCBF upon flexion compared with neutral position in $\mathrm{CCSCl}$ rat models at 4 wpi. Since the vasculature of the spinal cord is organized such that its ventral aspect and grey matter are supplied centrifugally by the central artery and the anterior part of the vasocorona, both of which arise from the ASA, ventral spinal cord compression caused by flexion position could probably exacerbate the ischaemic condition by compressing the ASA. We have previously reported that CSM patients with a "snake-eye" appearance of T2WI intramedullary hyperintensity suffered more severe flexion DSSEP deterioration in the clinic, probably caused by decreased SCBF at flexion.[12] Our LDF data directly indicated the perfusion loss in the compressed spinal cord during cervical flexion, as the studies mentioned above have suggested.

Finally, our data also indicated that motor functional loss was significantly related to decreased SCBF in $\mathrm{CCSCl}$ rat models at a flexed neck position. These findings are in general agreement with those of 
Bennett[55], who examined the effect of focal spinal cord ischaemia induced by segmental dorsal and ventral rhizotomy on MEPs and SSEPs in the cat spinal cord. He observed that MEPs more sensitively reflected spinal cord ischaemia than SSEPs but he did not measure SCBF. Fehlings et al.[36] reported that MEP amplitudes rather than SSEP amplitudes were significantly correlated with SCBF and injury severity (compression forces) in clip compression spinal cord injury rat models. Shine et al.[56] reported that MEPs disappeared prior to SSEPs after introducing ischaemia by aortic cross clamping; moreover, early MEP disappearance suggested a poor neurological outcome in patients undergoing thoracoabdominal aortic aneurysm repair. These animals and clinical studies indicated that compared with SSEPs, MEPs were more easily affected by spinal cord perfusion status. However, there are a number of other possible explanations for the high sensitivity of DMEPs to cervical flexion. For example, the corticospinal tract and the anterior horn motor neuronal system are mainly located at the ventral aspect of the spinal cord. Gooding et al. used dog models of cervical vascular insufficiency that were derived via selective vascular ligation to show that vascular disruption caused demyelination, glial fibrosis, and necrosis, especially in the corticospinal tracts.[57] Histological abnormalities in all dogs with combined compression and ischaemia were more pronounced than in dogs with either compression or ischaemia alone.[57] Thus, DMEP deterioration upon flexion in our $\mathrm{CCSCl}$ rat models was also more likely to be due to the combinatorial effects of ischaemia and direct neuronal compression.

\section{Conclusions}

In conclusion, the data from the present experiment show that changes in axonal conduction in the motor and somatosensory tracts of the spinal cord, as assessed by DMEPs and DSSEPs, were significantly related to the chronic compression time and neck positions. In addition, SCBF was significantly decreased after chronic compression, especially upon flexion, as demonstrated by dynamic MRI and dynamic LDF. Furthermore, spinal cord ischaemia may be intimately related to motor transduction dysfunction upon flexion in $\mathrm{CCSCl}$ models. Such a notion might open a new possible path for medical treatment for CSM, as opposed to the current practice that relies heavily on surgical decompression. Immobilization, such as that achieved by the use of cervical collars to prevent neck flexion in specific CSM patients, or drug treatment aiming to improve spinal cord perfusion may be effective in preventing the progression of functional loss.

\section{Abbreviations}

ASA: Anterior spinal artery; BBB: Basso, Beattie, and Bresnahan scale; IPT: inclined plane test; CCSCl: chronic compressive spinal cord injury; CSM: cervical spondylotic myelopathy; DMEPs: dynamic motor evoked potentials; DSSEPs: dynamic somatosensory evoked potentials; Hematoxylin and eosin (HE) staining; LDF: Laser Doppler flowmetry; MRI: Magnetic resonance imaging; RA-DMEP: relative amplitude of dynamic motor evoked potential; RA-DSSEP: relative amplitudes of dynamic somatosensory evoked potential; R-SCBF: relative change in spinal cord blood flow; SCBF: Spinal cord blood flow; T2WI: T2weighted image 


\section{Declarations}

\section{Ethics approval and consent to participate}

Animal experimental procedures were approved by the Animal Experimentation Ethics Committee and Institutional Animal Care and Use Committee (IACUC) at the First Affiliated Hospital of Sun Yat-sen University. The ethic number is: "[2020]458".

\section{Consent for publication}

All authors read and approved the final manuscript.

\section{Availability of data and materials}

The datasets generated during the current study are available from the corresponding author on reasonable request.

\section{Competing interests}

The authors declare that they have no competing interests.

\section{Funding}

The study was financially supported by the National Natural Science Foundation of China (No. 32071341), Natural Science Foundation of Guangdong Province (2017A030308004, 2020A1515010306) and Natural Science Foundation of Guangzhou City (201804020011).

\section{Authors' contributions}

$\mathrm{ZY}, \mathrm{XC}$ and $\mathrm{JC}$ were involved in performing animal experiments, analysis and interpretation of data, as well as in drafting and revising the manuscript. $X Z$ and $X P$ contributed to the conception and design of the study, and gave final approval of the version to be published. SL, HH, ZZ, FH, BC, YW, and SH were involved in acquisition of data. All authors read and approved the final manuscript.

\section{Acknowledgements}

We thank Prof. Yong Hu (Hong Kong University) for providing the spinal cord compression material. None of the authors have potential conflicts of interest to be disclosed.

\section{Supplementary Information}

The Supplementary Material for this article can be found online at: https://translationalneurodegeneration.biomedcentral.com/

\section{References}


1. Karadimas SK, Erwin WM, Ely CG, Dettori JR, Fehlings MG. Pathophysiology and Natural History of Cervical Spondylotic Myelopathy. Spine (Phila Pa 1976). 2013;38:21-36.

2. Joaquim AF, Baum GR, Tan LA, Riew KD. Dynamic Cord Compression Causing Cervical Myelopathy. (2586-6583 (Print)).

3. Jiang SD, Jiang LS, Dai LY. Degenerative cervical spondylolisthesis: a systematic review. Int Orthop. 2011;35(6):869-75.

4. Matsunaga S, Kukita M, Hayashi K, Shinkura R, Koriyama C, Sakou T, Komiya S. Pathogenesis of myelopathy in patients with ossification of the posterior longitudinal ligament. J Neurosurg. 2002;96(2 Suppl):168-72.

5. Fengbin Y, Deyu C, Xinwei W, Yu C, Jinhao M, Xinyuan L, Xiaowei L. Trauma-induced spinal cord injury in cervical spondylotic myelopathy with or without lower cervical instability. J Clin Neurosci. 2013;20(3):419-22.

6. Muhle C, Metzner J, Weinert D, Falliner A, Brinkmann G, Mehdorn MH, Heller M, Resnick D. Classification system based on kinematic MR imaging in cervical spondylitic myelopathy. AJNR Am J Neuroradiol. 1998;19(9):1763-71.

7. Kahle KT, Khanna A, Clapham DE, Woolf CJ. Therapeutic restoration of spinal inhibition via druggable enhancement of potassium-chloride cotransporter KCC2-mediated chloride extrusion in peripheral neuropathic pain. JAMA Neurol. 2014;71(5):640-5.

8. Breig A, Turnbull I, Hassler O. Effects of mechanical stresses on the spinal cord in cervical spondylosis. A study on fresh cadaver material. J Neurosurg. 1966;25(1):45-56.

9. Stamm S, McClellan JW 3rd, Knierim A, Suiter IP, Riew KD. Dynamic MRI Reveals Soft-Tissue Compression Causing Progressive Myelopathy in Postlaminectomy Patients: A Report of Three Cases. JBJS Case Connect. 2013;3(1):e17.

10. Morishita Y, Maeda T, Ueta T, Naito M, Shiba K. Dynamic somatosensory evoked potentials to determine electrophysiological effects on the spinal cord during cervical spine extension: clinical article. J Neurosurg Spine. 2013;19(3):288-92.

11. Qi Q, Huang S, Ling Z, Chen Y, Hu H, Zhan P, Zhang B, Zou X, Peng X. A New Diagnostic Medium for Cervical Spondylotic Myelopathy: Dynamic Somatosensory Evoked Potentials. World Neurosurg. 2020;133:e225-32.

12. Yu Z, Lin K, Chen J, Chen KH, Guo W, Dai Y, Chen Y, Zou X, Peng X. Magnetic resonance imaging and dynamic X-ray's correlations with dynamic electrophysiological findings in cervical spondylotic myelopathy: a retrospective cohort study. BMC Neurol. 2020;20(1):367.

13. Hirayama K, Tokumaru Y. Cervical dural sac and spinal cord in juvenile muscular atrophy of distal upper extremity. (0028-3878 (Print)).

14. Restuccia D, Rubino M, Valeriani M, Mirabella M, Sabatelli M, Tonali P. Cervical cord dysfunction during neck flexion in Hirayama's disease. Neurology. 2003;60(12):1980-3.

15. Fujimoto Y, Oka S, Tanaka N, Nishikawa K, Kawagoe H, Baba I. Pathophysiology and treatment for cervical flexion myelopathy. Eur Spine J. 2002;11(3):276-85. 
16. Izumida M. [A chronic spinal cord compression model in a rat with a 354A tumor]. Nihon Seikeigeka Gakkai Zasshi. 1995;69(10):977-91.

17. Yang T, Wu L, Wang H, Fang J, Yao N, Xu Y. Inflammation Level after Decompression Surgery for a Rat Model of Chronic Severe Spinal Cord Compression and Effects on Ischemia-Reperfusion Injury. Neurol Med Chir (Tokyo). 2015;55(7):578-86.

18. Kurokawa R, Murata H, Ogino M, Ueki K, Kim P. Altered blood flow distribution in the rat spinal cord under chronic compression. Spine (Phila Pa 1976). 2011;36(13):1006-9.

19. Hirai T, Uchida K, Nakajima H, Guerrero AR, Takeura N, Watanabe S, Sugita D, Yoshida A, Johnson $\mathrm{WE}, \mathrm{Baba} \mathrm{H}$. The prevalence and phenotype of activated microglia/macrophages within the spinal cord of the hyperostotic mouse (twy/twy) changes in response to chronic progressive spinal cord compression: implications for human cervical compressive myelopathy. PLoS One. 2013;8(5):e64528.

20. Kanchiku T, Taguchi T, Kaneko K, Yonemura H, Kawai S, Gondo T. A new rabbit model for the study on cervical compressive myelopathy. J Orthop Res. 2001;19(4):605-13.

21. Long HQ, Li GS, Lin EJ, Xie WH, Chen WL, Luk KD, Hu Y. Is the speed of chronic compression an important factor for chronic spinal cord injury rat model? Neurosci Lett. 2013;545:75-80.

22. Long HQ, Xie WH, Chen WL, Xie WL, Xu JH, Hu Y. Value of micro-CT for monitoring spinal microvascular changes after chronic spinal cord compression. Int J Mol Sci. 2014;15(7):12061-73.

23. Cheng X, Long H, Chen W, Xu J, Huang Y, Li F. Three-dimensional alteration of cervical anterior spinal artery and anterior radicular artery in rat model of chronic spinal cord compression by micro-CT. Neurosci Lett. 2015;606:106-12.

24. Long HQ, Li GS, Hu Y, Wen CY, Xie WH. HIF-1alpha/VEGF signaling pathway may play a dual role in secondary pathogenesis of cervical myelopathy. Med Hypotheses. 2012;79(1):82-4.

25. Cheng X, Long H, Chen W, Xu J, Wang X, Li F. The correlation between hypoxia-inducible factor1alpha, matrix metalloproteinase-9 and functional recovery following chronic spinal cord compression. Brain Res. 2019;1718:75-82.

26. Long HQ, Li GS, Cheng X, Xu JH, Li FB. Role of hypoxia-induced VEGF in blood-spinal cord barrier disruption in chronic spinal cord injury. Chin J Traumatol. 2015;18(5):293-5.

27. Xu J, Long H, Chen W, Cheng X, Yu H, Huang Y, Wang X, Li F. Ultrastructural Features of Neurovascular Units in a Rat Model of Chronic Compressive Spinal Cord Injury. Front Neuroanat. 2017;11:136.

28. Cheng X, Long H, Chen W, Xu J, Huang Y, Li F. Three-dimensional alteration of cervical anterior spinal artery and anterior radicular artery in rat model of chronic spinal cord compression by micro-CT. Neurosci Lett 2015(1872-7972 (Electronic)).

29. Basso DM, Beattie MS, Bresnahan JC. A sensitive and reliable locomotor rating scale for open field testing in rats. J Neurotrauma. 1995;12(1):1-21.

30. Rivlin AS, Tator $\mathrm{CH}$. Objective clinical assessment of motor function after experimental spinal cord injury in the rat. J Neurosurg. 1977;47(4):577-81. 
31. Puente N, Reguero L, Elezgarai I, Canduela MJ, Mendizabal-Zubiaga J, Ramos-Uriarte A, FernandezEspejo E, Grandes P. The transient receptor potential vanilloid-1 is localized at excitatory synapses in the mouse dentate gyrus. Brain Struct Funct. 2015;220(2):1187-94.

32. Dhillon RS, Parker J, Syed YA, Edgley S, Young A, Fawcett JW, Jeffery ND, Franklin RJ, Kotter MR. Axonal plasticity underpins the functional recovery following surgical decompression in a rat model of cervical spondylotic myelopathy. Acta neuropathologica communications. 2016;4(1):89.

33. de Haan P, Kalkman CJ. Spinal cord monitoring: somatosensory- and motor-evoked potentials. Anesthesiol Clin North Am. 2001;19(4):923-45.

34. Ueta T, Owen JH, Sugioka Y. Effects of compression on physiologic integrity of the spinal cord, on circulation, and clinical status in four different directions of compression: posterior, anterior, circumferential, and lateral. Spine (Phila Pa 1976). 1992;17(8 Suppl):217-26.

35. Fehlings MG, Tator $\mathrm{CH}$, Linden RD, Piper IR. Motor and somatosensory evoked potentials recorded from the rat. Electroencephalogr Clin Neurophysiol. 1988;69(1):65-78.

36. Fehlings MG, Tator $\mathrm{CH}$, Linden RD. The relationships among the severity of spinal cord injury, motor and somatosensory evoked potentials and spinal cord blood flow. Electroencephalogr Clin Neurophysiol. 1989;74(4):241-59.

37. Lee J, Satkunendrarajah K, Fehlings MG. Development and characterization of a novel rat model of cervical spondylotic myelopathy: the impact of chronic cord compression on clinical, neuroanatomical, and neurophysiological outcomes. J Neurotrauma. 2012;29(5):1012-27.

38. Hu Y, Wen CY, Li TH, Cheung MM, Wu EX, Luk KD. Somatosensory-evoked potentials as an indicator for the extent of ultrastructural damage of the spinal cord after chronic compressive injuries in a rat model. Clin Neurophysiol. 2011;122(7):1440-7.

39. Cheng X, Yu Z, Xu J, Quan D, Long H. Pathophysiological Changes and the Role of Notch-1 Activation After Decompression in a Compressive Spinal Cord Injury Rat Model. Front Neurosci 2021, 15(46).

40. Wang J, Rong W, Hu X, Liu X, Jiang L, Ma Y, Dang G, Liu Z, Wei F. Hyaluronan tetrasaccharide in the cerebrospinal fluid is associated with self-repair of rats after chronic spinal cord compression. Neuroscience. 2012;210:467-80.

41. Coughlin DJ, Rymarczuk GN, Dirks MS. Noncalcified Hypertrophic Ligamentum Flavum Causing Severe Cervical Stenosis and Myelopathy: Case Report and Review of the Literature. World Neurosurg. 2016;95:618. e621-618 e626.

42. Zeitoun D, El Hajj F, Sariali E, Catonné Y, Pascal-Moussellard H. Evaluation of spinal cord compression and hyperintense intramedullary lesions on T2-weighted sequences in patients with cervical spondylotic myelopathy using flexion-extension MRI protocol. The Spine Journal. 2015;15(4):668-74.

43. Zhang L, Zeitoun D, Rangel A, Lazennec JY, Catonné Y, Pascal-Moussellard H. Preoperative Evaluation of the Cervical Spondylotic Myelopathy With Flexion-Extension Magnetic Resonance Imaging. Spine (Phila Pa 1976). 2011;36(17):E1134-9. 
44. Weidauer S, Nichtweiss M, Hattingen E, Berkefeld J. Spinal cord ischemia: aetiology, clinical syndromes and imaging features. Neuroradiology. 2015;57(3):241-57.

45. Suri A, Chabbra RP, Mehta VS, Gaikwad S, Pandey RM. Effect of intramedullary signal changes on the surgical outcome of patients with cervical spondylotic myelopathy. The spine journal: official journal of the North American Spine Society. 2003;3(1):33-45.

46. Chen CJ, Lyu RK, Lee ST, Wong YC, Wang LJ. Intramedullary high signal intensity on T2-weighted MR images in cervical spondylotic myelopathy: prediction of prognosis with type of intensity. Radiology. 2001;221(3):789-94.

47. You JY, Lee JW, Lee E, Lee GY, Yeom JS, Kang HS. MR Classification System Based on Axial Images for Cervical Compressive Myelopathy. Radiology. 2015;276(2):553-61.

48. Weidauer S, Nichtweiss M, Lanfermann $\mathrm{H}$, Zanella FE. Spinal cord infarction: MR imaging and clinical features in 16 cases. Neuroradiology. 2002;44(10):851-7.

49. Cheng X, Xu J, Yu Z, Xu J, Long H. LncRNA Xist Contributes to Endogenous Neurological Repair After Chronic Compressive Spinal Cord Injury by Promoting Angiogenesis Through the miR-32-5p/Notch-1 Axis. Front Cell Dev Biol. 2020;8:744.

50. Olive JL, McCully KK, Dudley GA. Blood flow response in individuals with incomplete spinal cord injuries. Spinal Cord. 2002;40(12):639-45.

51. Yamada T, Morimoto T, Nakase H, Hirabayashi H, Hiramatsu K, Sakaki T. Spinal cord blood flow and pathophysiological changes after transient spinal cord ischemia in cats. Neurosurgery. 1998;42(3):626-34.

52. Jing Y, Bai F, Chen H, Dong H. Using Laser Doppler Imaging and Monitoring to Analyze Spinal Cord Microcirculation in Rat. J Vis Exp 2018(135).

53. Brain WR, Knight GC, Bull JW. Discussion of rupture of the intervertebral disc in the cervical region. Proc R Soc Med. 1948;41(8):509-16.

54. Karadimas SK, Moon ES, Yu WR, Satkunendrarajah K, Kallitsis JK, Gatzounis G, Fehlings MG. A novel experimental model of cervical spondylotic myelopathy (CSM) to facilitate translational research. Neurobiol Dis. 2013;54:43-58.

55. Bennett MH. Effects of compression and ischemia on spinal cord evoked potentials. Exp Neurol. 1983;80(3):508-19.

56. Shine TS, Harrison BA, De Ruyter ML, Crook JE, Heckman M, Daube JR, Stapelfeldt WH, Cherry KJ, Gloviczki P, Bower TC, et al. Motor and somatosensory evoked potentials: their role in predicting spinal cord ischemia in patients undergoing thoracoabdominal aortic aneurysm repair with regional lumbar epidural cooling. Anesthesiology. 2008;108(4):580-7.

57. Gooding MR, Wilson CB, Hoff JT. Experimental cervical myelopathy. Effects of ischemia and compression of the canine cervical spinal cord. J Neurosurg. 1975;43(1):9-17.

\section{Figures}


a.

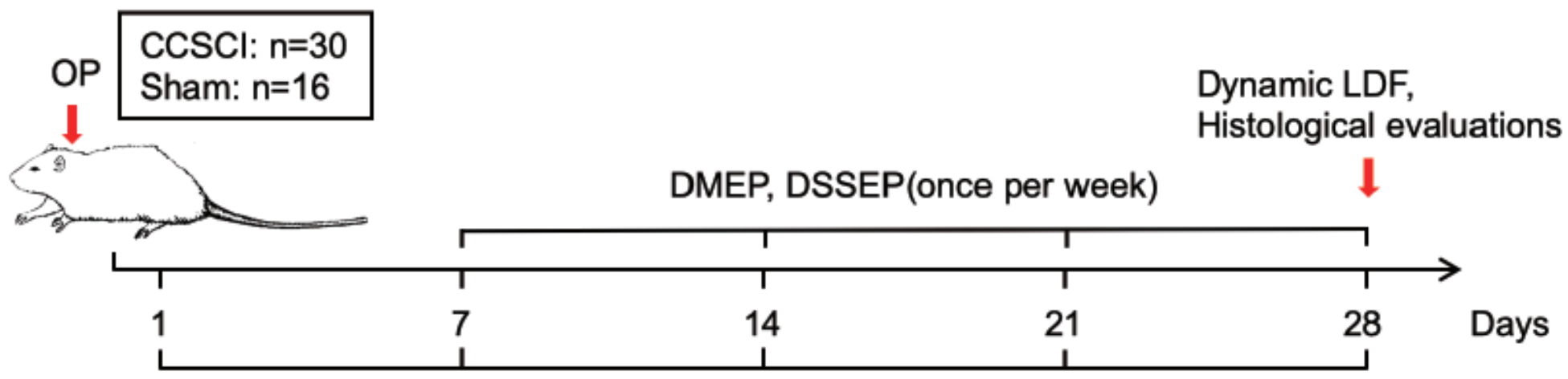

BBB, IPT(1 day, 1, 2, 3, 4 weeks post-surgery)
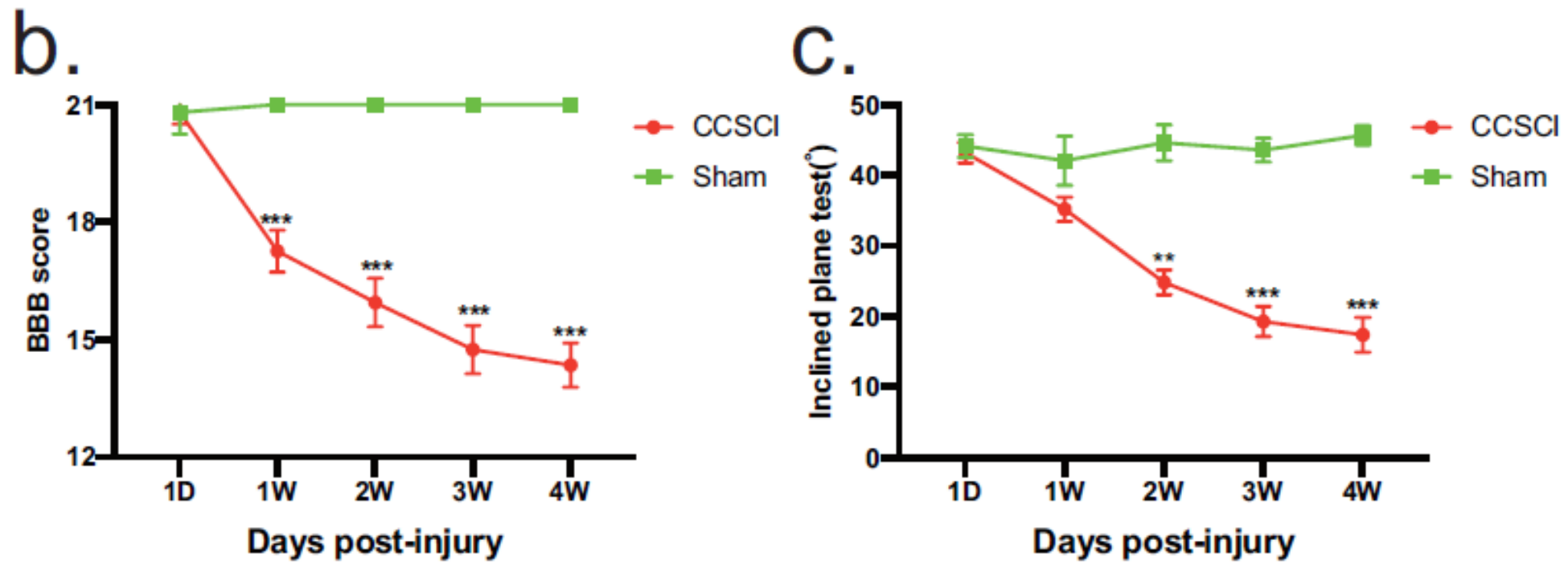

Figure 1

Progressive neurological dysfunction of the rats with chronic compressive spinal cord injury (CCSCl). (a) Experimental design. (b) Animals were tested weekly starting from $1 \mathrm{dpi}$ in an open field, and motor function recovery was evaluated according to the BBB score. (c) Animals were tested weekly starting from 1 dpi using the IPT, and the angle $\left({ }^{\circ}\right)$ was recorded to reveal motor function recovery. ${ }^{*} \mathrm{p}<0.01$, $\star \star * p<0.001$ 

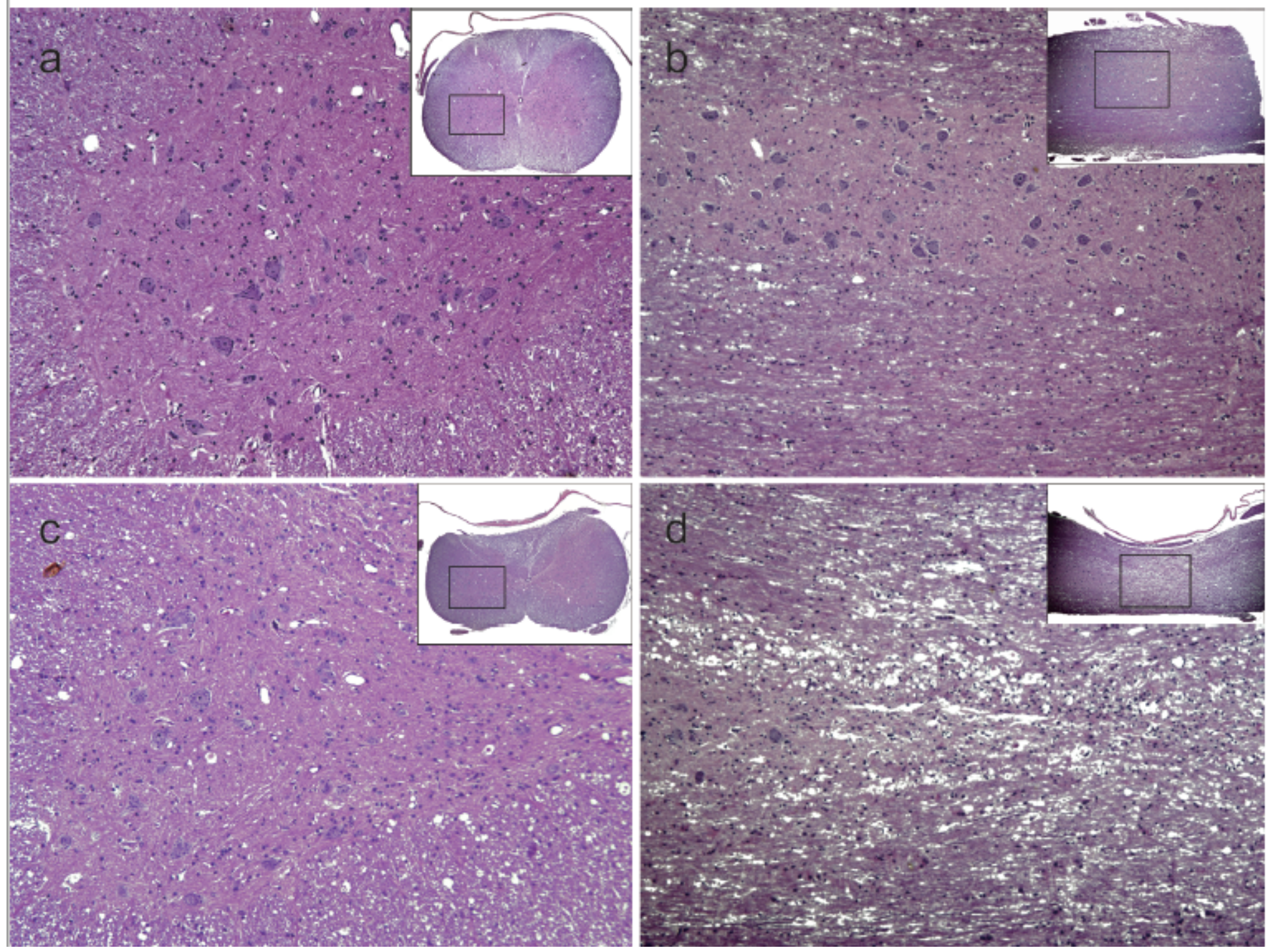

\section{Figure 2}

HE staining of cervical spinal cord from Sham and CCSCl rats $(a, b)$ The spinal cord was intact in the sham group. The polygonal Nissl bodies inside neurons in the grey matter anterior horn were large and dense. (c) In a transverse section of a CCSCl model, the compressed spinal cord exhibited structural damage, including fragmentation of neuronal nuclei, pyknosis, neuropil damage, degradation of the extracellular matrix, interstitial edema, cytoplasmic reduction, and cavity formation. The dorsal funiculi suffered the most severe breakage. The neuronal cell bodies in the anterior horn were round and small. Nissl body chromatolysis were shown inside the neurons. The insults of the cord circulation in a $\mathrm{CCSCl}$ model including central canal enlargement and intra-tissue bleeding in the grey matter. (d) The CCSCl models' longitudinal neurological conduction pathways were severely disrupted, as neuronal loss in the gray and axon breakage in the white matter were observed in sagittal sections. 


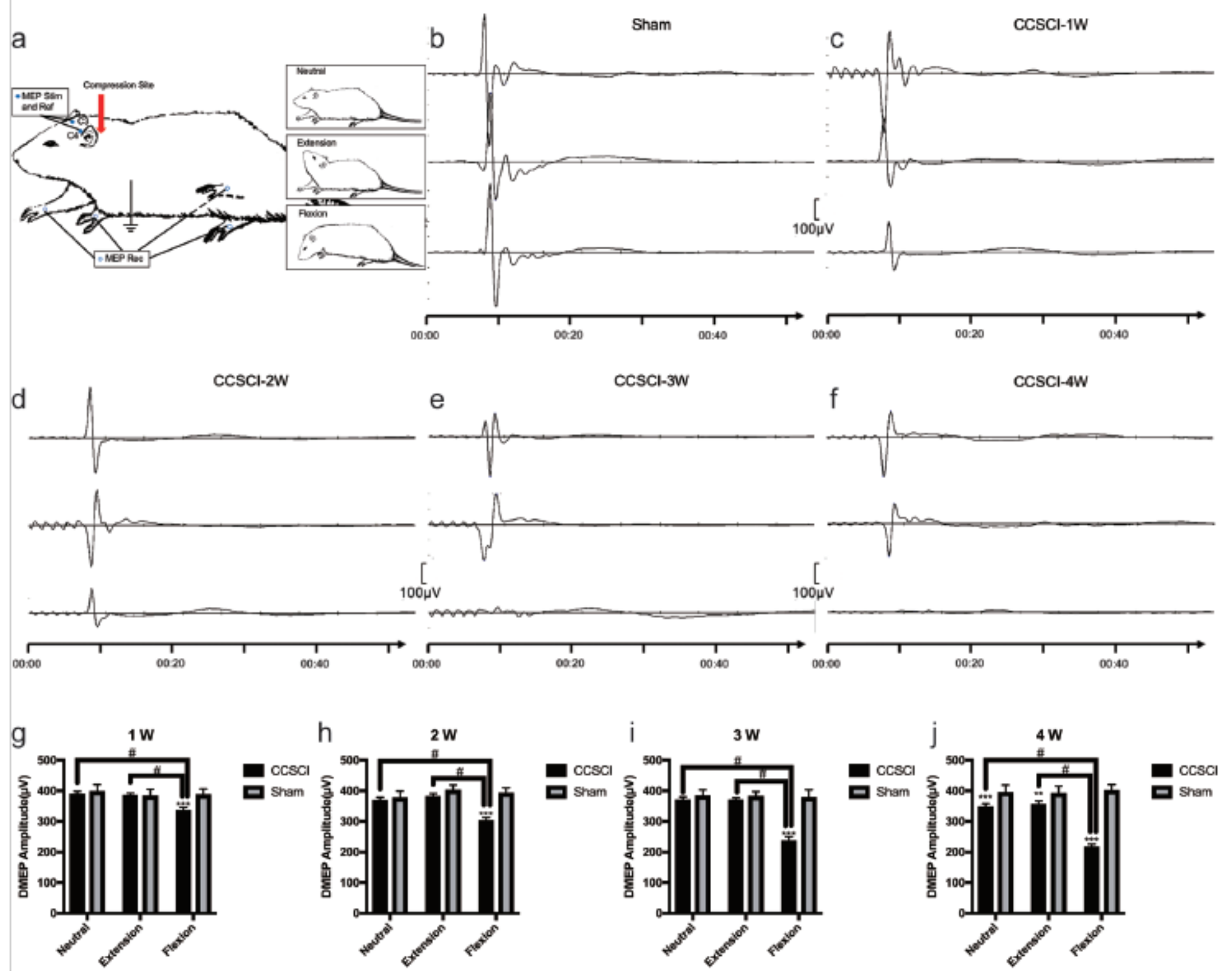

Figure 3

Dynamic motor evoked potentials (DMEPs) illustrating. (a) Electrode placement. To elicit the MEP, anodal stimuli (Stim) and a reference (Ref) electrodes were applied to the skull (C3 and C4). The responses were recorded from electrodes on the extensor digitorum communis in the forelimb and tibialis anterior in the hindlimb. (b) Representative images showing the N1 DMEPs in a sham group rat. The upper, middle and lower waves were DMEPs performed upon neutral, extension and flexion positions respectively. (c-f) Representative images showing the N1 DMEPs in different time points of the CCSCl model rats. The N1 waves of representative $\mathrm{CCSCl}$ rats at 3 and 4 weeks post-surgery upon flexion were abolished $(\mathrm{e}, \mathrm{f}) .(\mathrm{g}-\mathrm{k})$ Quantification of DMEP amplitudes in CCSCl and sham groups at each time point. Amplitudes for abolished N1 waves were excluded. The DMEPs did not change at all neck positions after 10 minutes of implanting the unexpanded compression material. The DMEP amplitudes in CCSCl group did not change upon neutral and extension until 4 weeks post injury compared with the sham group. Upon flexion, the DMEPs in the $\mathrm{CCSCl}$ group deteriorated significantly compared with the other two neck positions, also with the flexion DMEPs in the sham group. **, *** Indicated statistical significance $(p<0.01,0.001$ 
respectively) in the difference between the Sham and $\mathrm{CCSCl}$ group using t test. \# Indicated statistical significance $(p<0.05)$ in the difference between various neck positions MEP responses using one-way ANOVA and post hoc test.

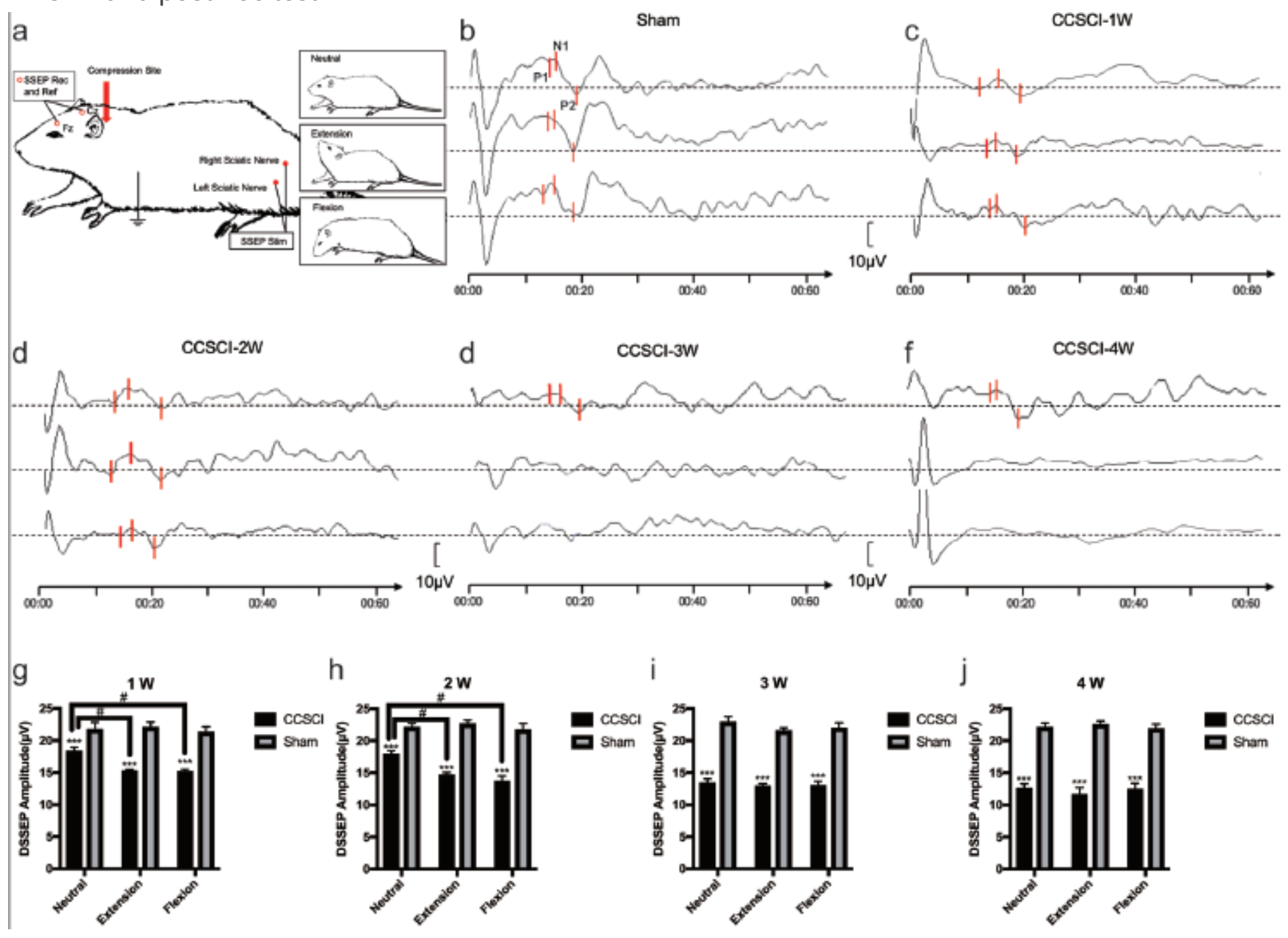

Figure 4

Dynamic somatosensory evoked potentials (DSSEPs) illustrating. (a) Electrode placement. To elicit the SSEPs, stimuli (Stim) were applied to the sciatic nerve and recorded from the $\mathrm{Fz}$ and $\mathrm{Cz}$ points on the skull. (b) Representative images showing the DSSEP waves in a sham group rat. The upper, middle and lower waves were DSSEPs performed upon neutral, extension and flexion positions respectively. (c-f) Representative images showing the DSSEPs in different time points of the CCSCl model rats. P1, N1, P2 peaks on each wave were marked with three bars respectively. The amplitude of each wave was defined as the peak-to-peak amplitude of the N1 and P2 waves. The waves of representative CCSCI rats at 3 and 4 weeks post-surgery upon extension and flexion were abolished $(e, f)$. $(g-k)$ Quantification of DSSEP amplitudes in $\mathrm{CCSCl}$ and sham groups at each time point. Amplitudes for abolished waves were excluded. The latency and amplitude of DMEPs peaks in the sham group upon all three positions remained unchanged at all testing time points. From 1 to 4 weeks of chronic compression injury, increasing numbers of rats showed abolished SSEP with the increase of compression time. The CCSCI 
models had more abolished DSSEP waves and significantly lower DSSEP amplitude than the sham models upon all neck positions at each time point after injury (t-test, $p<0.001$ ). At the first 2 wpi, the $\mathrm{CCSCl}$ rats had less abolished waves and significantly higher (ANOVA post hoc test, $p<0.05$ ) DSSEP amplitude upon neutral compared with the other two neck positions. The DSSEP amplitudes at all 3 neck positions were similar after 3 wpi. ${ }^{* \star}$, $* \star \star$ Indicated statistical significance $(p<0.01,0.001$ respectively) in the difference between the Sham and $\mathrm{CCSCl}$ group using t test. \# Indicated statistical significance $(\mathrm{p}<$ $0.05)$ in the difference between various neck positions MEP responses using one-way ANOVA and post hoc test.
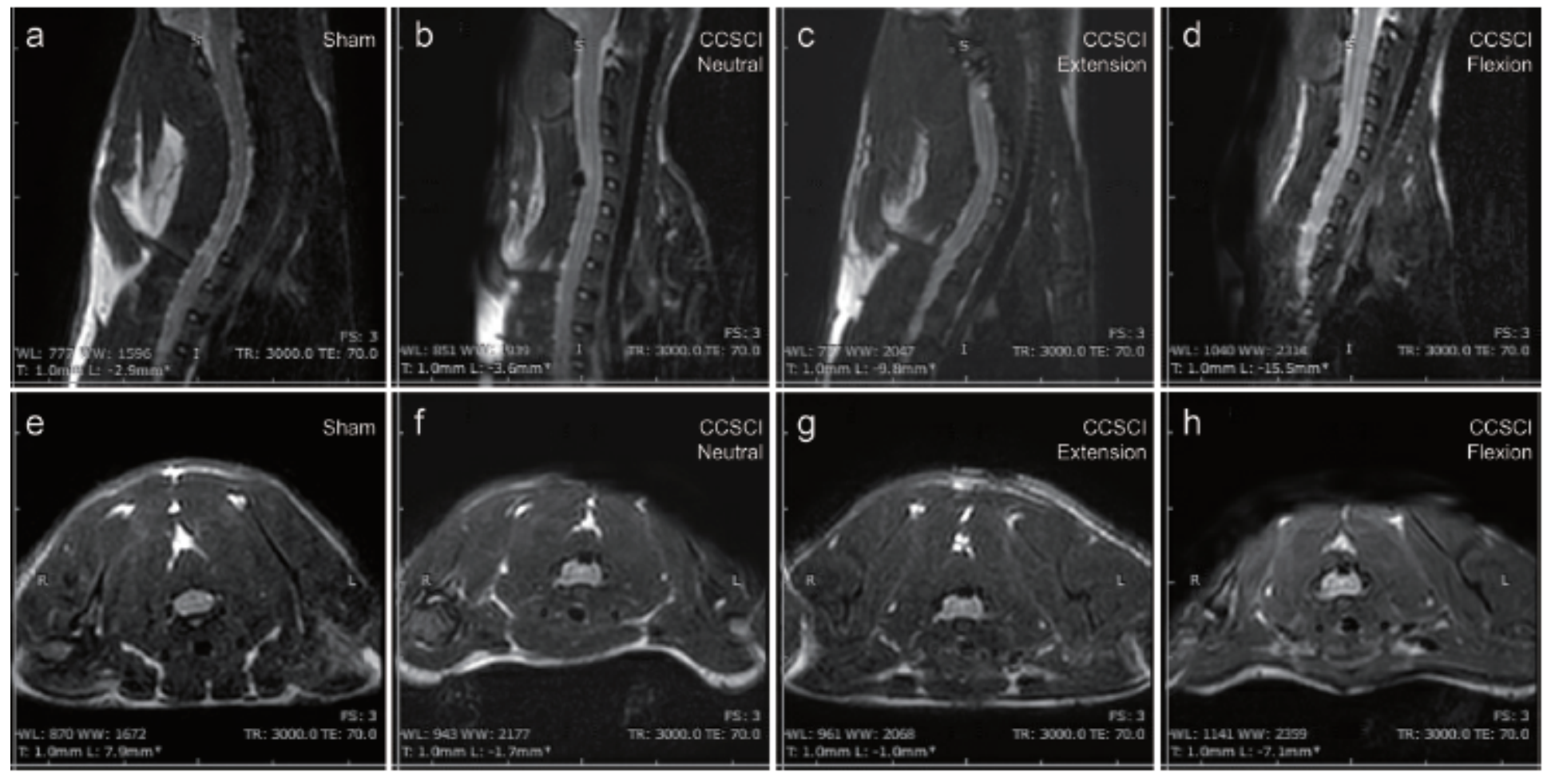
i

\section{Sagittal MRI}

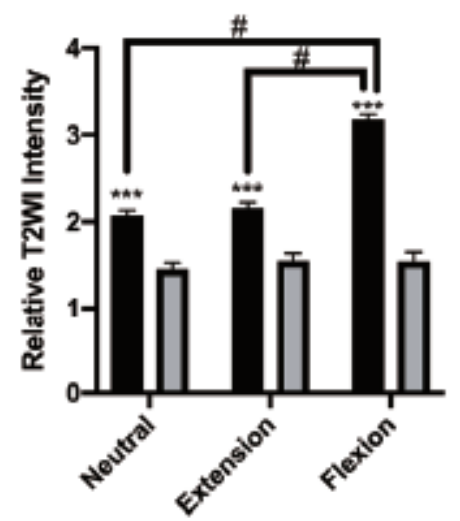

$\mathrm{CCSCl}$

Sham

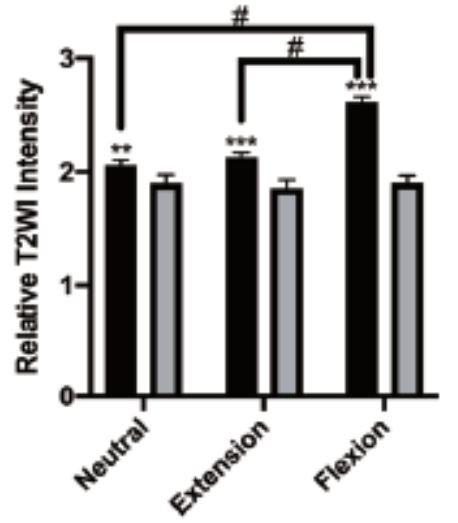

$\operatorname{ccscl}$

Sham

\section{Figure 5}

The signal intensity changes of MR images in different positions after CCSCl. (a, e) Representative images showing neutral position of a rat in the sham group. There was no material compressing the spinal cord. $(b, f)$ Representative images showing neutral position of a CCSCl rat model. The compression material is localized on the posterior side at the $\mathrm{C} 5 / 6$ level of the cord. There existed a significantly higher 
T2WI contrast medium intensity at the injured site upon neutral position of a CCSCl rat compared with a sham model. (c, g) Representative images showing extension position of a CCSCl rat model. $(d, h)$

Representative images showing flexion position of a $\mathrm{CCSCl}$ rat model. Upon flexion, the T2WI signals of the cord at both sagittal and transverse sections were significantly more intense than that upon neutral or extension positions. ${ }^{*}, * *, * * *$ Indicated statistical significance $(p<0.05,0.01,0.001$ respectively) in the difference between the Sham and CCSCl group using $t$ test. \# Indicated statistical significance $(p<0.05)$ in the difference between various neck positions MEP responses using one-way ANOVA and post hoc test.

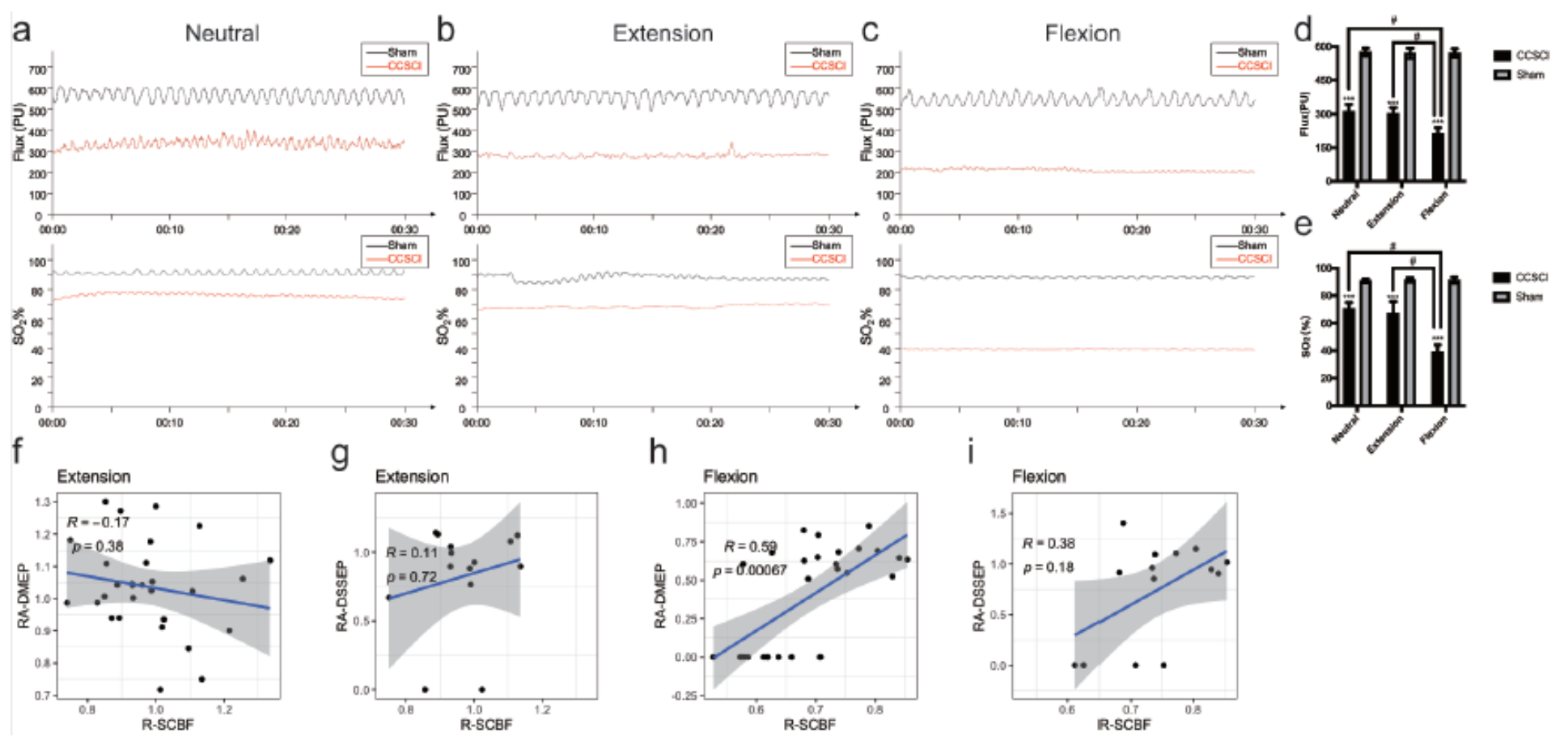

Figure 6

The alteration of instant spinal cord blood flow and oxygen saturation level in different positions after $\mathrm{CCSCl}$, and its correlations with electrophysiological changes. The maps of instant blood flow and oxygen saturation level were evaluated by using a laser Doppler flowmetry at the C5/ 6 segment of the cervical cords upon neutral (a), extension (b) and flexion (c) positions in Sham and CCSCl groups. Spinal cord blood flow (SCBF) (d) and oxygen saturations (e) at the compression sites of the CCSCl rat models were significantly lower than that of sham models at all three neck positions $(p<0.001)$. Both of them also deteriorated significantly upon flexion $(p<0.001)$. Spearman correlation analysis demonstrating that the R-SCBF was not correlated with relative amplitude of DMEPs (RA-DMEP) or relative amplitude of DSSEPs (RA-DSSEP) upon extension $(f, g)$. It significantly correlated with the RA-DMEP (Spearman correlation, $\mathrm{R}=0.72, \mathrm{p}<0.001)(\mathrm{h})$, but not with RA-DSSEP upon flexion (i). * $, * \star, * \star *$ Indicated statistical significance ( $<0.05,0.01,0.001$ respectively) in the difference between the Sham and CCSCl group using t test. \# Indicated statistical significance $(p<0.05)$ in the difference between various neck positions MEP responses using one-way ANOVA and post hoc test.

\section{Supplementary Files}


This is a list of supplementary files associated with this preprint. Click to download.

- 4.Additionalfile1.docx 